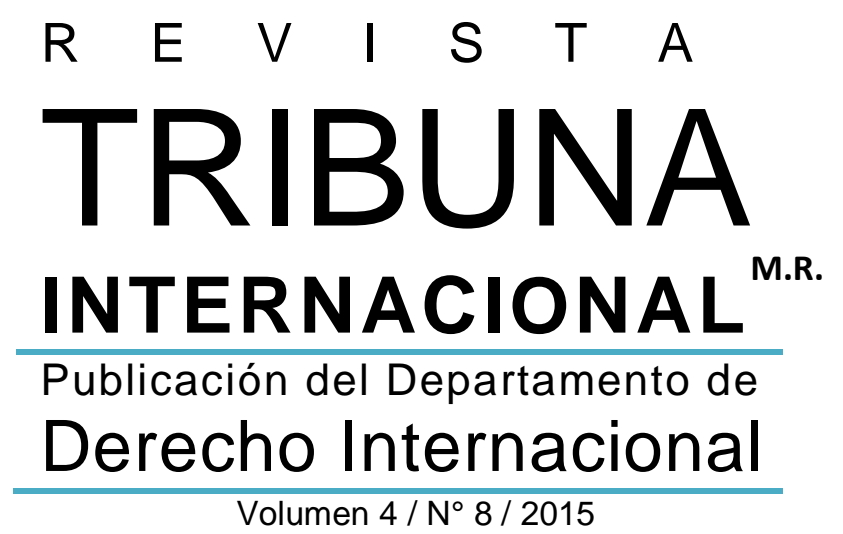

FACULTAD DE DERECHO 


\section{Rector de la Universidad de Chile}

Ennio Vivaldi Véjar

Av. Alameda Libertador Bernardo O'Higgins 1058, Santiago

\section{Representante legal}

Davor Harasic Yaksic

Decano de la Facultad de Derecho

Universidad de Chile

\section{Director Departamento Derecho Internacional}

Edmundo Vargas Carreño

\section{Director (S) Revista Tribuna Internacional}

Mario Arnello Romo

\section{Editor General Revista Tribuna Internacional}

Luis Valentín Ferrada Walker

\section{Comité Editorial}

Íñigo Álvarez Gálvez (Universidad de Chile, Chile)

Gonzalo Aguilar (Universidad de Talca, Chile)

José Carlos Fernández Rosas (Universidad

Complutense de Madrid, España)

Claudio Grossman (American University, EE.UU.)

Mattias Kumm (New York University, EE.UU.)

Hugo Llanos (Universidad Central, Chile)

Cecilia Medina (Universidad Diego Portales, Chile)

Elina Mereminskaya (Universidad de Chile, Chile)

Mónica Pinto (Universidad de Buenos Aires, Argentina)

\section{Fundador de la Revista Tribuna Internacional}

Mario Ramírez Necochea $†$

\section{Revista Tribuna Internacional M.R.}

Publicación del Departamento de Derecho Internacional de la Facultad de Derecho de la Universidad de Chile. Su objetivo es fomentar la reflexión, el debate, el análisis y la comunicación sobre el derecho internacional en forma pluralista y con rigor científico. Se publica cada semestre en los meses de junio y diciembre mediante convocatoria abierta a la publicación de artículos y monografías inéditos, comentarios de jurisprudencia, recensiones y comentarios de libros, en los campos de derecho internacional público y privado, derecho internacional de los derechos humanos y relaciones internacionales, tanto en castellano como inglés.

La Revista Tribuna Internacional fue creada por Decreto Exento No 8.466 de la Rectoría de la Universidad de Chile, de 22 de marzo del 2011

Volumen 4/ No 8 / 2015

www.tribunainternacional.uchile.cl ISSN 0719-482X (versión en línea)

Departamento de Derecho Internacional Facultad de Derecho Universidad de Chile Av. Santa María 076, $4^{\circ}$ piso Providencia, Santiago de Chile

\section{Diseño y producción:}

Facultad de Derecho

Universidad de Chile

Se autoriza la reproducción total o parcial del contenido de la publicación, siempre que se reconozca y cite el/ la/ los/ las autor/a/es/as y la publicación, no se realicen modificaciones a la obra y no se la utilice para fines comerciales. 


\title{
Cambio Climático en Estados Unidos: el caso Utility Air Regulatory Group v. Environmental Protecction Agency
}

\author{
Climate Change in United States: the case Utility Air Regulatory Group v. \\ Environmental Protecction Agency
}

\section{Andrea Lucas Garín}

andrealucas@hcla.uni-heidelberg.de

Doctora en Derecho y Ciencias Sociales, Abogada y Especialista en Derecho Público, Universidad Nacional de Córdoba. Máster en Derecho Internacional, Universidad de Heidelberg y Universidad de Chile. Coordinadora Académica, Heidelberg Center para América Latina - Universidad de Heidelberg. Profesora de la Universidad Alberto Hurtado y del Instituto de Estudios Internacionales.

Resumen: El comentario presenta un breve panorama de cómo se ha enfrentado al fenómeno de Cambio Climático en los Estados Unidos de Norteamérica, se analiza el precedente jurisprudencial más relevante decidido por la Corte Suprema de Estados Unidos (Massachusetts v. EPA) en el año 2007 y se revisan las particularidades que conlleva el caso Utility Air Regulatory Group v. EPA, del 2004, para alcanzar algunas conclusiones que ameritan la situación doméstica e internacional.

Palabras claves: Cambio Climático - Derecho Medioambiental - Derecho estadounidense Corte Suprema de los Estados Unidos.

Abstract: The review presents a brief overview of how United States of America have faced the phenomenon of Climate Change, the most relevant precedent decided by the Supreme Court of the United States (Massachusetts v. EPA) in 2007 is analyzed and besides a review of the particularities that involved the case Utility Air Regulatory Group v. EPA, in 2014, to reach some conclusions which considers domestic and international situation.

Key words: Climate Change - Environmental Law - American Law - Supreme Court of the United States. 


\section{Palabras previas}

El Cambio Climático ${ }^{1}$ se percibe como un gran desafío ambiental, el más importante del siglo XXI, y su regulación se presenta como una verdadera tarea por hacer, tanto para la comunidad internacional, los bloques regionales y cada uno de los Estados.

El Sistema de Protección Internacional del Cambio Climático, encabezado por la Convención Marco de Naciones Unidas sobre el Cambio Climático (CMNUCC), concentra los esfuerzos de los Estados por estabilizar los Gases de Efecto Invernadero (GEI). Junto con el Protocolo de Kioto, constituyen el régimen internacional con el cual la comunidad internacional afronta el calentamiento global.

Estados Unidos de Norteamérica ha estado en el centro del debate de Cambio Climático muchas veces, principalmente por no haber adherido al Protocolo de Kioto. Este instrumento derivado de la CMNUCC, fue negociado durante más de dos años y adoptado en la conferencia celebrada en Japón, en 1997. Para algunos constituye el más importante acuerdo multilateral negociado hasta la fecha (al menos hasta antes de los acuerdos alcanzados en diciembre del 2015 en la Conferencia de París).

Dentro del régimen internacional de Cambio Climático, las negociaciones han estado concentradas en la buscar un acuerdo para un instrumento que reemplace al Protocolo de Kioto una vez que fenezca el segundo período de compromisos, desde el 2013 al 2020. Las previsiones indican que las nuevas obligaciones de reducción de GEI serán compartidas por países desarrollados y en desarrollo, y el resultado de la Conferencia de París será en definitiva el nuevo marco para abordar este tema ${ }^{2}$.

En el ámbito de las activas negociaciones de Cambio Climático es interesante conocer qué ocurre con Estados Unidos, por lo que presentaremos un breve panorama acerca de cómo se ha enfrentado al fenómeno este influyente país, analizaremos el precedente jurisprudencial más relevante decidido por la Corte Suprema de Estados Unidos en el año 2007 y revisaremos las particularidades que nos trae el caso Utility Air Regulatory Group v. EPA, del 2014; todo lo que

1 El calentamiento generado por el aumento de la radiación solar relacionado con la emisión de Gases de Efecto Invernadero desde la revolución industrial, determinó que la contaminación aumentara y con ello sus efectos. Al inicio de la discusión del fenómeno del Cambio Climático había escépticos respecto al origen antropocéntrico o entre aquellos que pensaban que el planeta sólo estaba bajo presión. La labor continuada del Panel Intergubernamental del Cambio Climático ha sido fundamental en disipar estos interrogantes y corroborar el origen antropocéntrico del fenómeno. A fines del año pasado el Panel Intergubernamental renovó la base científica del fenómeno de Cambio Climático cuando brindó su Quinto Reporte. Aseguró que " la influencia humana en el sistema climático es clara y las recientes emisiones antropógenas del Cambio Climático son las más altas de la historia”. Vid. Intergovernmental Panel on Climate CHANGE (IPPC), Quinto Informe de Evaluación, 2014 [en línea] $<$ http://www.ipcc.ch/news_and_events/docs/ar5/ar5_syr_headlines_es.pdf > [consulta: 20.dic.2015].

2 La $21^{\text {a }}$ Conferencia de las Partes de la Convención Marco de Naciones Unidas sobre el Cambio Climático de 2015 (COP21/CMP11), también llamada "París 2015" se desarrolló en París entre el 30 de noviembre y el 11 de diciembre de 2015. 
Revista Tribuna Internacional

Volumen $4 \cdot \mathrm{N}^{\text {o }} 8 \cdot 2015 \cdot$ pp. $283-291$

ISSN 0719-482X (versión en línea)

nos hace prever que se emprenderán nuevas acciones a nivel de doméstico y de la comunidad internacional.

El caso que vamos a analizar nos permite observar en toda su magnitud que la problemática ambiental envuelve una confrontación entre Derechos Fundamentales y la regulación en post del cuidado ambiental, con todo un abanico de consecuencias que aquí asume la Corte Suprema de Estados Unidos. Posiblemente, como la regulación ambiental implica limitaciones de Derechos Fundamentales, en Estados Unidos es el Poder Judicial que se erige como árbitro constante de estas medidas.

\section{Introducción al escenario estadounidense de Cambio Climático}

Es evidente que la postura de Estados Unidos en relación con el régimen internacional de Cambio Climático ha sido errática. Primero ratificó la CMNUCC, suscrita en la Conferencia de Naciones Unidas sobre Medio Ambiente y Desarrollo, llevada a cabo en Río de Janeiro en 1992, integrando su Anexo I. Luego, negoció el Protocolo de Kioto, pero en 1997 se aprobó en el Senado de Estados Unidos la Resolución Byrd-Hagel, que lo rechazaba ${ }^{3}$. No obstante ello, el Presidente Clinton lo suscribió en 1998, pero enseguida no lo envió al Congreso (a pesar de haber un cambio de postura más accesible a las negociaciones con este Presidente). Finalmente, la Administración Bush rechazó el Protocolo directamente ${ }^{4}$.

Así, por una parte no se ratificó el Protocolo de Kioto y, por otra, durante el gobierno del Presidente Bush se renunció, en el año 2001, a continuar con las negociaciones al respecto 5 . Recién en la Conferencia de las Partes de Bali, en el año 2007, Estados Unidos decidió volver a las negociaciones para avanzar en sus compromisos sobre el Cambio Climático.

Tradicionalmente este país ha generado el veinticinco por ciento $(25 \%)$ de las emisiones totales de dióxido de carbono 6 , por lo que su adhesión y cumplimiento al Protocolo era vital para lograr que este entrara en vigencia en el período de 1997 al 2005. En definitiva, fue Rusia la

3 “Una respuesta directa al cambio de política sobre Cambio Climático de la administración Clinton fue la resolución del Senado 98, patrocinado por los Senadores Byrd y Hagel, que fue aprobada 95-0 en julio de 1997. Esta resolución llamada 'Byrd-Hagel' dejó la ratificación legislativa de un protocolo con objetivos y calendarios vinculantes, condicionada a los compromisos vinculantes simultáneos para los países en desarrollo". La traducción nos pertenece. SCHRÖDER, Heike. Negotiating the Kyoto Protocol. An analysis of negotiation dynamics in international negotiations. Hamburg, LIT, 2001, p. 37.

4 Cfr. Hufbauer, Gary et al. Global Warming and the World Trading System. Washington, Peterson Institute for Internationals Economics, 2009, p. 2.

5 Hunter, Salzman y Zaelke han expresado que si bien Estados Unidos no es parte del Protocolo de Kioto, y por ende, no participa de las negociaciones post-Kioto, ha acordado participar de un 'diálogo de cooperación a largo plazo', siempre y cuando el diálogo no se base en reducciones obligatorias. HuNTER, David et al. International Environmental Law and Policy. $3^{a}$ edición, Saint Paul (Minnesota), Foundation Press, 2007, p. 727.

6 La EPA informa que al 2011 los Estados Unidos emitían un 16\% de GEI a nivel global. Vid. EPA. "Global Greenhouse Gas Emissions Data. Emissions by Country", [en 2015, línea] <http://epa.gov/climatechange/ghgemissions/global.html\#four> [consulta: 20.dic.2015]. 
que, con ratificación al Protocolo en el año 2004 y considerando su nivel de sus emisiones, permitió que este entrara en vigor ${ }^{7}$.

Si bien Estados Unidos no ha ratificado el Protocolo, ello no significa que tal país no tenga una política sobre Cambio Climático, como a veces se ha creído. En especial a nivel estadual, tanto California como otros estados poseen una posición activa al respecto, registrándose asimismo en estos últimos años varios proyectos de ley en el Congreso estadounidense.

La Agencia de Protección Ambiental de los Estados Unidos (EPA, por sus siglas en inglés) informa respecto a algunas iniciativas de regulación en el marco de la Ley del Aire Limpio y sobre varias medidas para abordar el fenómeno, tal como la recopilación de datos de emisiones, programas voluntarios de reducciones de emisiones de GEI, etc.; también informa que se encuentra evaluando políticas más globales, las que hasta ahora no han sido aprobadas por el Congreso ${ }^{8}$.

Los elementos básicos de la política climática de Estados Unidos son resumidos por Hufbauer, Charnovitz y Kim en tres puntos: subsidios al etanol, estándares energéticos y políticas climáticas de los estados federados, en algunos casos regionales ${ }^{9}$. Justamente los estándares energéticos son el objeto del caso judicial que motiva este comentario.

Las medidas emprendidas para afrontar el Cambio Climático se inscriben en el marco de la Ley de Aire Limpio. Esta normativa, vigente desde 1970 y con varias enmiendas, permite a la EPA ocuparse de todo lo relativo a contaminantes del aire en orden a la protección de la salud y bienestar de la población.

Como parte de la Ley de Aire Limpio, se cuenta con el Programa de Lluvia Ácida de los Estados Unidos, que se considera ha sido un antecedente relevante y la inspiración del Sistema de Comercio de Emisiones de la Unión Europea, verdadero modelo a nivel mundial de instrumento para afrontar la problemática climática ${ }^{10}$.

Dado que Estados Unidos es un Estado federal, hay competencias en materia ambiental que conservan los estados. Por ello, a nivel estadual se han aplicado diversos mecanismos de reducción de GEI. Suele mencionarse como ejemplo a "Massachusetts, que se convirtió en el primer estado de Estados Unidos en imponer límites a la emisión de CO2 en las antiguas

Seguimos a Halvorssen, Anita. "The Kyoto Protocol and Developing Countries - the Clean Development Mechanism", en Colorado Journal of International Environmental Law and Policy, vol. 16, N 2, 2005, pp. 362-363.

8 Vid. la página web de EPA, [en línea] < http://epa.gov/espanol/cambioclimatico/actividadesEPA.html> [consulta: 20.dic.2015].

9 Hufbauer et al., Global Warming..., op.cit., p. 4. Los autores también realizan un raconto de los proyectos de ley presentados para afrontar el problema del Cambio Climático. Vid. pp. 23-29.

10 El Sistema EU ETS (European Union Emission Trading Scheme) se ubica en la Directiva 2003/87/CE del Parlamento Europeo y del Consejo, de 13 de octubre de 2003, por la que se establece un régimen para el comercio de derechos de emisión de gases de efecto invernadero en la Comunidad y por la que se modifica la Directiva 96/61/CE del Consejo. Vid. EuR-LEX, [en línea] <http://eur-lex.europa.eu/legal-content/ES/TXT/HTML/?uri=CELEX:02003L008720140430\&from $=\mathrm{EN}>$ [consulta: 20.dic.2015]. 
Revista Tribuna Internacional

Volumen $4 \cdot \mathrm{N}^{\text {o }} 8 \cdot 2015 \cdot$ pp. $283-291$

ISSN 0719-482X (versión en línea)

plantas térmicas de energía" ${ }^{\text {11 }}$. Este estado ha fijado límites obligatorios para seis instalaciones estatales, con la finalidad de reducir sus emisiones en $10 \%$ en el año 2006 respecto al año base promedio de 1997-1999.

El Estado de California se ha erigido como el líder en la protección ambiental y en la eficiencia energética, aplicando desde 2006 un programa obligatorio para limitar las emisiones de GEI. La Ley de Soluciones al Calentamiento Global de ese año ${ }^{12}$, incluye metas de emisión de carbono iguales a las de 1990 para alcanzarse en el año 2020. Contiene asimismo un programa de comercio de emisiones (cap and trade programme) que está vigente desde inicios de $2012^{13}$.

La Presidencia de Obama ha planteado el Cambio Climático como una prioridad, con la intención de lograr niveles de emisión iguales al año 1990 para el año $2020^{14}$. En agosto de 2015 se hizo público el Plan de Energía Limpia con el cual se pretende reducir la polución generada por las plantas de energía, plan elaborado por la EPA ${ }^{15}$.

\section{El camino planteado por Massachusetts v. Environmental Protection Agency}

En los Estados Unidos, parte de la estrategia para enfrentar el Cambio Climático a nivel federal ha pasado por el desarrollo jurisprudencial, a diferencia de otros países donde los Poderes Legislativos y Ejecutivos son quienes toman las iniciativas para enfrentar el fenómeno.

Debe recordarse que la Suprema Corte de los Estados Unidos en el caso Massachusetts v. EPA dispuso que la acción de la Agencia no estaba en conformidad con la Ley de Aire Limpio, la cual autorizaba a la oficina federal a regular las emisiones de los vehículos de motor nuevos en función de su posible impacto en el Cambio Climático. La sentencia fue decidida el 2 de abril de $2007^{16}$.

11 COMisión ECONÓmiCa para América LATINA (CEPAL). El mercado de carbono en América Latina y el Caribe: balance y perspectivas. Santiago, Naciones Unidas, 2004 p. 20. El estudio de CEPAL comenta la Oregon Climate Trust que es un fondo para proyectos de reducciones de nuevas plantas de generación eléctrica con metas de reducción de emisiones de CO2, habiendo sido la ley estadual promulgada en 1997.

12 La Ley 32, en inglés Global Warming Solutions Act, Assembly Bill (AB) 32, se puede consultar en español, [en línea] <http://www.arb.ca.gov/cc/ab32/en_espanol.htm> [Consulta: 20.dic.2015].

13 Según relata QIN, Tianbao. "The Emissions Trading System in the Context of Climate Change: China’s Response”, en Ruppel, Oliver C., Roschmann, Christian, Ruppel-SchliCHTing, Katharina (editores). Climate Change: International Law and Global Governance. Baden-Baden (Alemania), Nomos, 2013, vol. I, p. 471.

14 Esto explican Torney, Diarmuid y Kamal Gueye, Moustapha. "Climate Change Mitigation Policies in Selected OECD Countries: Trade and Development Implications for Developing Countries", en ICTSD Series on Climate Change and Competitiveness, No 8, p. 10, [en línea] < http://www.ictsd.org/themes/climate-and-energy/research/climate-changemitigation-policies-in-selected-oecd-countries $>$ [Consulta: 20.dic.2015].

15 Mayores informaciones en website de EPA (2015).

16 La cita legal es 549 U.S. 497 (2007). 
En Massachusetts, el máximo tribunal sostuvo que los gases de efecto invernadero están comprendidos en la definición general del término "contaminante del aire" de la Ley de Aire Limpio, y que por ello la EPA estaba facultada y obligada a regular las emisiones de gases de efecto invernadero procedentes de fuentes móviles (como los automóviles y camiones). Había una omisión por parte de la Agencia que la Corte Suprema condenó, una inacción en la regulación de los GEI necesaria para combatir el Cambio Climático, omisión que también era condenada internacionalmente, ante el nivel de emisiones tradicionales de un país tan relevante como los Estados Unidos.

Cabe señalar que, en respuesta a Massachusetts, la EPA promulgó las normas de emisiones de GEI para los nuevos vehículos de motor y estableció las fuentes fijas sujetas a un programa especial (PSD y el Título V), que incluía las fuentes sobre la base de su potencial de emitir gases de efecto invernadero.

Estos dos antecedentes jurisprudenciales también nos permiten ejemplificar cómo el Poder Judicial puede colaborar en la lucha contra por el Cambio Climático, comprometido con la temática ambiental.

\section{Particularidades del caso Utility Air Regulatory Group v. Environmental Protection Agency}

Las partes litigantes son el Utility Air Regulatory Group, la American Chemistry Council, la Energy Intensive Manufacturers Working Group on Greenhouse Gas Regulation, la Southeastern Legal Foundation Inc., la Chamber of Commerce of the United States y el Estado de Texas, todos contra la EPA ${ }^{17}$.

El medio jurisdiccional que utiliza la Corte Suprema de los Estados Unidos es el Writ of Certiorari, que permite al máximo tribunal seleccionar los casos que atiende, atento la trascendencia que generan en el sistema jurídico estadounidense.

Como breve descripción del caso, podemos decir que fue introducido por las empresas y los consorcios que litigan contra la EPA en orden a la delimitación de los alcances de normas para las emisiones de "gases de efecto invernadero". La cuestión de derecho que aborda la Corte Suprema es si la Agencia está autorizada a determinar extensivamente en sus reglamentos límites de emisiones de GEI (fuentes fijas), conforme la Ley del Aire Limpio. La sentencia fue emitida el 23 de junio de 2014.

17 La cita del caso es la siguiente: Utility Air Regulatory Group v. Environmental Protection Agency et al. Certiorari to the United States Court of Appeals for the District of Columbia Circuit, No. 12-1146, 573 U. S. (2014). 
Revista Tribuna Internacional

Volumen $4 \cdot \mathrm{N}^{\mathrm{o}} 8 \cdot 2015 \cdot$ pp. $283-291$

ISSN 0719-482X (versión en línea)

Respecto a la Ley de Aire Limpio, la sentencia señala que esta impone requisitos de permisos en las fuentes fijas, tales como fábricas y centrales eléctricas. A su vez, existe una programa federal para la "Prevención de Deterioro Significativo" (PSD), cuyas disposiciones hacen que sea necesario contar con permiso para modificar las estructuras de las principales fuentes emisoras (fuentes con el potencial de emitir 250 toneladas por año de "cualquiera de los contaminantes del aire" o 100 toneladas por año para ciertos tipos de fuentes). Estas fuentes, para obtener permiso, deben cumplir con las limitaciones de emisiones que reflejan la "mejor tecnología de control disponible". La norma considera que es ilegal operar cualquier "fuente importante", dondequiera que se encuentre, sin un permiso. Una "fuente importante" es una fuente estacionaria con el potencial de emitir 100 toneladas por año de cualquiera de los contaminantes del aire. Por ejemplo, lo serían shoppings, edificios y escuelas.

El listado de contaminantes considerados por la Agencia como "gases de efecto invernadero", es decir, sustancias que contribuyen al "“cambio climático global", incluye, según el Manual de la Ley de Aire Limpio, los siguientes seis contaminantes: dióxido de azufre, material particulado (PM), dióxido de nitrógeno, monóxido de carbono, ozono y plomo. Varias de estas sustancias están también comprendidas en el Protocolo de Kioto ${ }^{18}$.

El razonamiento jurídico utilizado para la resolución del caso por parte de la Corte Suprema considera que la EPA ha excedido su autoridad legal cuando interpreta la Ley de Aire Limpio (por el programa PSD y el Título V) al exigir permisos para fuentes fijas en función de sus emisiones de gases de efecto invernadero de manera extensiva.

En la sentencia Utility se incluyen referencias al proceso abierto al público de elaboración de normas por $\mathrm{EPA}^{19}$ que se siguió luego del caso Massachusetts. Incluso se indica que la propia Agencia ha reconocido que las exigencias de permisos para todas las fuentes de emisiones de GEI podrían implicar exceder los umbrales legales y, de este modo, por decisiones administrativas, ampliar radicalmente los programas y los sujetos obligados por la ley federal.

Lo que resulta destacable en el fallo es que el Poder Judicial se erige en controlador de la actividad administrativa aún cuando esta actividad hace referencia a informaciones científicas y muy técnicas que seguramente han sido atendidas por EPA en el proceso abierto de elaboración de las normas objeto de la controversia. El control judicial de la actividad administrativa se reactiva y conlleva que el poder administrador revise la efectividad de sus procesos de toma de decisiones. La sentencia hace mención a las consecuencias económicas de la regulación realizada por EPA.

18 El Anexo A del Protocolo de Kioto señala como gases de efecto invernadero al Dióxido de carbono (CO2), el Metano (CH4), el Óxido nitroso (N2O), los Hidrofluorocarbonos (HFC), los Perfluorocarbonos (PFC) y el Hexafluoruro de azufre (SF6). En el 2012 se generó la Enmienda de Doha al Protocolo de Kioto, que contiene un segundo período de compromisos, desde el 2013 al 2020, junto con un nuevo GEI, el Trifluoruro de nitrógeno (NF3), que se suma al listado del Protocolo.

19 Los procesos de elaboración de normas ambientales usualmente incluyen la consulta al público y este es un reconocido instrumento de gestión ambiental utilizado por las organizaciones administrativas a cargo de la misma. 
En el pronunciamiento no hay referencias al Sistema de Protección Internacional de Cambio Climático ni a las exigencias multilaterales al respecto. Los alcances sólo son nacionales. Esto es llamativo en atención a la importancia que tienen las medidas unilaterales tomadas por tan importante actor de la comunidad internacional. En esta sentencia, la Corte Suprema reconfirma las facultades de la EPA para regular la emisión de GEI y vuelve a abordar a nivel federal la lucha contra el Cambio Climático.

\section{Algunas conclusiones}

La regulación del Cambio Climático genera numerosos desafíos, que complejiza la tarea estatal, en este caso de la EPA, la que debe dar respuestas tanto a grupos de la industria y de los Estados como a grupos ambientalistas que buscan regulaciones más estrictas.

Cabe señalar que Estados Unidos tiene una tradición de conciencia ambiental e incluso a inicios de los años sesenta y los setenta, la comunidad nacional financió la investigación y el conocimiento sobre Cambio Climático, pasando luego, en los ochenta, a ayudar a la expansión del tema y a que se ubicara en la agenda internacional, en palabras de Schröder ${ }^{20}$.

El fenómeno jurídico que se genera en torno a la litigación ambiental y el rol rector que tienen las sentencias en determinar la política doméstica para enfrentar el Cambio Climático, es algo digno de apreciar. En ningún lugar la litigación ambiental ha alcanzado la importancia que tiene en Estados Unidos.

Pareciera que en ese país se están intentando superar las críticas que consideraban que no tenía un paquete de leyes federales sobre Cambio Climático que se ocupara de manera integral de la reducción de emisiones a través de todo el país ${ }^{21}$.

Decisiones judiciales como Massachusetts y Utility mantienen a la Corte Suprema como un actor que seguirá participando de la toma de decisiones en materia climática, tanto para llamar la atención por las omisiones de la EPA como para revisar su actuación.

La política federal sobre Cambio Climático de los Estados Unidos sin dudas traerá consecuencias sobre el comercio mundial, en razón de su relevancia comercial. Mantener a los Estados Unidos participando de las negociaciones multilaterales debe ser una preocupación

SCHRÖDER, Negotiating the Kyoto Protocol..., op.cit., p. 34.

21 Esta era la opinión de Francesco Síndico, vid. SindiCO, Francesco. "Climate and Trade in a Divided World: Can Measures adopted in the North end up Shaping Climate Change Legislative Frameworks in the South?”, en RICHARDSON, Benjamin J., Le Bouthillier, Yves, MCLeOD-Kilmurray, Heather y WoOD, Stepan (editores). Climate Law and Developing Countries. Legal and Policy Challenges for the World Economy. Cheltenham (Reino Unido), Edward Elgar, 2009, p. 364. 
Revista Tribuna Internacional

Volumen $4 \cdot \mathrm{N}^{\text {o }} 8 \cdot 2015 \cdot$ pp. $283-291$

ISSN 0719-482X (versión en línea)

para el Sistema de Protección Internacional de Cambio Climático de modo de evitar las tan temidas decisiones unilaterales que sólo obstaculizarían el libre comercio.

La complejidad del fenómeno climático nos permite concluir que la litigación no es el único camino para abordar la gestión de riesgos adecuada que haga frente al Cambio Climático; es bueno contar con un Poder Judicial comprometido con la temática, pero que de ninguna manera reemplace la toma de medidas que el Poder Legislativo y el Poder Ejecutivo deben realizar. De otro modo, la sociedad estadounidense y, la sociedad global por las características del fenómeno, no verán aumentada su resiliencia. 